\title{
Barriers to Males Involvement in Family Planning Services in Arba Minch Town, Southern Ethiopia: Qualitative Case Study
}

\author{
Alemu Tamiso ${ }^{1}$, Admasu Tassew ${ }^{2}$, Henok Bekele ${ }^{3}$, Zale Zemede ${ }^{1}$, Admasu Dulla ${ }^{1}$ \\ ${ }^{1}$ Department of Public health/Unit of epidemiology and biostatistics, College of Medicine and Health Sciences, \\ Arba Minch University, Arba Minch, Ethiopia \\ ${ }^{2}$ Department of Biology, College of Natural science, Arba Minch University, Arba Minch, Ethiopia \\ ${ }^{3}$ Department of Planning, Adare Hospital, Southern Region Health Bureau, Hawassa, Ethiopia
}

\begin{tabular}{l}
\hline \hline Article Info \\
\hline Article history: \\
Received Nov 22, 2015 \\
Revised Dec 21, 2015 \\
Accepted Jan 16, 2016 \\
\hline
\end{tabular}

\section{Keyword:}

Arba Minch

Barriers

Family planning

Male involvement

\begin{abstract}
Effective utilization of family planning services are major contributors of improved maternal health. This, in turn, needs adequate involvement of male partners through direct uptake the services and approval of the spouses' usage. Yet the family planning method utilization as well as male involvement is indicated to be low in Ethiopia. Qualitative case study was conducted in August 2015 in Arba Minch town to identify barriers to male involvement in uptake of family planning services. Open ended, semistructured questionnaire was used to interview the participants. Four men and four women are purposively selected. Interviews were conducted in audibly private location after verbal consent was obtained from the recruited individuals. All interviews were recorded and transcribed verbatim. The narratives were then translated to English and the responses are aggregated in to nine concepts. The final aggregated data were analyzed using OpenCode 3.4 software based on thematic framework analysis. Total of eight participants (four men and four women) were interviewed where seven are married and one is single. Participants identified that, Perception (or opinion) towards family planning as women's issue, Sex preference for inheritance and considering children as measure of blessing of and Fear of partner sexual promiscuity and to reduce women's attractiveness were barriers for male involvement in modern family planning method. Future interventions aimed at improving family planning services and methods utilization in Arba Minch town and similar settings need to consider these bottlenecks for male involvement.
\end{abstract}

Copyright () 2016 Institute of Advanced Engineering and Science. All rights reserved.

\section{Corresponding Author:}

Alemu Tamiso,

Department Public Health,

College of Medicine and Health Sciences,

Arba Minch University, Southern Ethiopia.

Email: alemutamiso@yahoo.com

\section{INTRODUCTION}

Family planning services are integral in the prevention of unwanted pregnancy, reduction of unsafe abortions, and promotion of childbirth spacing. The services, in turn, need to assure effective involvement of men to better achieve their intended goals. Several studies show that men's involvement has positive impact on uptake of family planning services [1]-[4]. However, family planning services traditionally targeted women regardless of the fact that men's involvement is equally important and childbearing has an impact on men's life too. A review of male involvement in decision making in family planning in Sub-Saharan Africa reflected low involvement of men in family planning programs [5]. A research report from Muslim communities in Ghana suggested further low male involvement in family planning uptake and higher non- 
approval of for spouses contrary to perfect knowledge and awareness to contraceptive options [6]. Similarly, low males' involvement was reported from rural districts of Uganda, Debremarkos town and Bahir Dar city in Ethiopia [7]-[9].

Barriers to males' involvement took varying forms based on the context of the communities considered. Program for Appropriate Technology in Health (PATH) [10] documented number of factors. Women and children targeted nature of the services, limited number of male contraceptives available, rumors and misinformation concerning the effects of the available options and unfavorable social or religious climate are to mention some. In India and Nepal, many men resist vasectomy due to myths that the procedure causes physical weakness or impotence and reduces a man's capability to provide for his household. In parts of Nepal, on the other hand, men disapprove their wives to use contraceptives because they believe that it may make the women promiscuous [3]. A study in Kenya identified mere attitude or negative perceptions towards family planning by men and the belief that it's woman's affair as main barriers [1]. Another study from Nyanza Province in Kenya on HIV-Positive Men indicated that concerns about side effects of contraceptives, lack of knowledge about contraceptive methods, myths and misconceptions including fear of infertility, and lack of male focus in family planning methods and service delivery as key barriers to male involvement [11].

Barriers to male involvement in family planning in Ethiopia have similarities to the reports from elsewhere. However, lack of information and low level of education got considerable share. Studies conducted in the north, north-west, south-west and south central parts of the country imply lack of appropriate information, inaccessibility of family planning services, attitudes of men towards family planning, perceived fear of side-effects, cultural or religious oppositions and desire for more children are among main barriers of male involvement in family planning in Ethiopia [2],[8],[9],[12],[13].

Despite few quantitative research works undertaken on male involvement in family planning in the country as aforementioned, in-depth understanding of the rationale for men's low participation has been underexplored. Ochako et al. [14] and Kabagenyi et al. [7] showed that awareness and knowledge to family planning services often do not translate to use, hence it necessitates undertaking an in-depth qualitative investigation to come up with working solution to improve male involvement. To address this gap, this study examines level of male involvement in family planning services utilization and associated factors.

\section{RESEARCH METHOD}

\subsection{Study area}

The study area was Arba Micnh town, Gamo Gofa Zone Southern Ethiopia $505 \mathrm{~km}$ south west of the country's capital Addis Ababa and $273 \mathrm{~km}$ south of the regional capital Hawassa. According to national population and housing census in 2007 , the town was inhabited by 74,843 people (39,192 male and 35, 651 female) [15]. The current projected population of estimated to be over 100,000 residents. There are one general hospital, two public health centers and number of private clinics of varying rank rendering services to the health needs of the residents. Family planning services are mainly obtained from the public health institutions.

\subsection{Study design}

A qualitative phenomenology study was conducted using in-depth interview with four women and four men of reproductive age in Arba Minch town, Southern Ethiopia. Semi-structured questionnaire was used for the interview with emphasis on preferred family size, the role of husbands in the family with regard to family planning, knowledge about contraceptives and male involvement in family planning.

\subsection{Participant selection}

Study participants were purposively selected of whom seven were married and one is unmarried who were living in Arba Minch town at the time of the study. The participants were composed of five family planning service clients (two men and three women) living in the town and three service providing personnel (two men and a woman) in Arba Minch general hospital. Informed verbal consent was taken from the participants prior to data collection where they are eligible to join the study.

\subsection{Data collection}

Open ended, semi-structured question guides were used to explore perceptions regarding barriers to male involvement in family planning service utilization. Interviews were conducted in Amharic language and in audibly private areas until saturation was reached. All of the study participants were encouraged to openly discuss their opinions. 


\subsection{Data analysis}

All interviews were recorded and transcribed verbatim. After validating the transcription, the typed narratives were then translated into English and verified for accuracy. The English translated narrative per individual respondents was aggregated into 22 concepts, later pooled to 9 before fed into software. Data were analyzed using OpenCode 3.4 software. Finally, thematic analysis with quotation was used to report the output.

\section{RESULTS AND ANALYSIS}

In-depth interview was conducted on eight individuals, four females and four males of which all were married, but one. Five of the participants were protestant, two were orthodox, one was Islam followers and their age interval ranges from 21 to 38 years. The average duration of the interview was about 27 minutes. Redundancy of idea was checked and only three the participants' responses were considered for the final analysis. Analysis of the final selected data using Open Code software yielded eight categories and twenty-two codes which were relevant with participant's information. Accordingly, four main categories were identified as a barrier to male's involvement in contraceptive utilization: i) Influence of culture \& religion, ii) Perception (or opinion) towards family planning as women's issue, iii) Sex preference for inheritance and considering children as measure of blessing of and iv) Fear of partner sexual promiscuity and to reduce women's attractiveness.

\subsection{Influence of culture \& religion}

All participants believe that religion has an adverse influence on family planning service utilization. Religious husbands are said to less likely utilize family planning services themselves and they disapprove utilization by their wives as well. The underlying cause of their decision is the belief that having many children is the God's blessing. A 38 years old participant who is father of four children states this fact as follows: "Yes, religion adversely influences family planning utilization. For instance, in my religion children are considered as God's gift and they discourage contraceptive usage for family planning. Even though majority of believers do not agree with this idea, some extremist believers do not use family planning services. They simply bear 8, 9 or up to 12. The astonishing fact is that even those who sustain their life by fire wood collection bear 10 or 12. Sometimes I try to challenge them to use the family planning services, but they refuse claiming their children are God's gifts and He will sustain them".

Cultural norms are also stated to play similar negative role in family planning service utilization as indicated by a participant who is mother of three children. She blamed the cultural customs of the area where she was brought up as an obstacle to uptake family planning in general and permanent family planning methods in particular. "For instance, in rural areas if a women takes contraceptives some time before giving first birth, her husband will follow another younger girl. This is because it is custom in some places to have more than one wife. That is a bad influence of culture. Therefore, the wife prefers to bear child without her choice just for the sake of securing her husband. Child bearing women are even respected in some areas. This can be another reason why women may not consider permanent family planning methods."

Accordingly, the custom allows a man can marry to more than one woman for the sake of having children and women who bear more children are respected. This frees the man to loosely attend family planning matters and forces the wives to bear more children to stabilize their family and claim better social status in the community. As those rural areas mentioned by the participant are in the vicinity of Arba Minch town, the opinion of those residents migrated to the town from the nearby rural areas do not vary from people who are bound to the customs aforementioned.

\subsection{Perception of family planning as women's issue}

Three of the participants believed that it is only women's business to use family planning methods because of social norms and impact of family planning on men. Some of them merely participate in discussion. Although some husbands allow their wives to use contraceptives, they themselves are much less willing to use family planning methods for men. The perception that only female should use family planning services is composed of intricate reasoning including lack of knowledge, refusal of wives for men condom, and misconception that vasectomy may terminate the manly nature of the husband at all. One participant claims that men have to use family planning services as women did, but he himself does not actually use. "I know that, but I don't use it. I have a story again. There was a neighbor who is father of five daughters and three sons. They have no knowledge of other family planning options. The person being enough with the children he has proposed to his wife to use condom. However, the wife refused thinking it may slip out inside her body. They quarreled much until I was called to mediate them on the issue. Concerning the other option (vasectomy), I heard another story. There was a boy residing with a white (foreigner). He used to meet the 
housemaid residing together and make her pregnant frequently. Learning this event the white man might have injected him something. Now the guy is employee in our university, but people talk of him as he has been castrated by that white man. He currently has no child at all. People even talk of him that he has no manly feelings to women. Therefore, I don't want to use that."

\subsection{Sex preference for inheritance and considering children as measure of blessing}

Because of cultural and social values most people prefer son to daughter to lead the family and inherit their possessions in case when the family head dies. Accordingly, one male participant underlined that sex preference was the main barrier to male's involvement in contraceptive utilization. He explained the experience he came across as follows: "I had a neighbor who already fathered 7 daughters. He was protestant extremist. But when he thought it as if his wife's problem, he changed his religion to marry to another woman in search of bearing a baby boy. However, the new wife gave birth to additional two daughters. Now with advice from friends including myself he returned back to his family and faith. This story I'm telling you is very recent one".

Others claimed that people particularly the parents of the married couples belief that children are measure of blessing of God. Three of the four client women to family planning services told that parents extremely discourage contraceptive utilization at all. When this disapproval comes with religious husbands it is implied as one important obstacle to male involvement in family planning services.

\subsection{Fear of partner sexual promiscuity and to reduce women's attractiveness}

Men perceive that wives become calm and submit to their husbands when they bear children. A young woman participant who regularly follows family planning services put this fact on her own words. "Some husbands believe that a wife become calm and submits to her husband. They believe a woman settles when she bears a child."

Women's utilization of family planning services is also perceived to lead them to become unfaithful and reflect women's intentions to avoid pregnancy within extramarital sexual relationships. Therefore, perceived sexual promiscuity is one important barriers male involvement in allowing their wives to utilize family planning methods.

Family planning methods were also described as enhancing women's physical attractiveness by delaying or preventing childbearing, which made men reluctant to support women's contraceptive use. A woman participant explains how this perception affects family planning service utilization: "There are some rude husbands who are envious on the beauty of their wives. They think their wives beauty won't drag attention of other men as it fades if they bear children frequently. Because of this silly reason they let them simply bear children. But I don't agree with that; it's character that dictates the loyalty of a woman not the number of children she bears".

\section{DISCUSSION}

The findings of this study show that male involvement in family planning method and service utilization is low. Moreover, they often disapprove the utilization of family planning services by their wives based on several barriers. These findings are in line with the reports by others researchers who conducted cross-sectional survey in different parts of Ethiopia [8],[9],[13].

The low male involvement was attributed to men's reluctance to support use of modern contraceptive methods for their spouses or themselves. This is in turn based on cultural and religion influence, Sex preference for inheritance and considering children as measure of blessing of God, perception that family planning is a women's issue, lack of knowledge and fear sexual promiscuity by contraceptive using wives. Qualitative study using focused group discussion in two rural districts and a separate study using key informants in Kiyeyi of Tororo district in Uganda came up with similar findings [7],[16]. Two independent researches in different parts of Kenya also reported similar outcomes [11],[14]. The awareness and knowledge to family planning services and methods was not so poor among the participant males in this study. Their practical involvement does not translate their awareness in the contrary as indicated in similar study by Ochako [14].

However, there are some points specific to this study which are not well explored by other studies such as the intention of attempt to degrade the beauty of wives through childbearing to secure loyalty, and the fact that bearing more children gives a better social status. The effect of parents of married couples in religious family settings can also be pointed out as particular to the current. 


\section{CONCLUSION}

The current study identified influence of culture and religion, perception towards family planning as women's issue, sex preference for inheritance and considering children as measure of blessing of God, and fear of partner sexual promiscuity as major barriers to male involvement in family planning services utilization. Future interventions aimed at improving family planning services and methods utilization in Arba Minch town and similar settings need to consider these points identified as main bottlenecks for male involvement. Creating awareness on benefits of family planning and fostering family planning options with detail merits and demerits of each is also of crucial importance. Emphasis must be given to male contraceptive methods and males friendly family planning services with appropriate counseling. We also recommend further qualitative research especially through focused group discussion to weed out possible individualistic views and to assess the wider perspective of the community at large. As one of the participants indicated that rural based parenting of the married couples is still evident in their perception further qualitative study must be conducted in the rural settings in the vicinity of the town.

\section{ACKNOWLEDGEMENTS}

We would like to express our kind gratitude to all the participants who actively devoted their time to come up with such an invaluable data. We would like also to give our gratitude to Arba Minch University, college of Medicine and Health Science, for giving opportunity to conduct this qualitative research.

\section{REFERENCES}

[1] Muiga WM., "Factors Influencing Male Participation In Family Planning: A Case of Kutus Township In Kirinyaga County In Kenya”, MA Thesis, University of Nairobi, 2013.

[2] Tilahun, T., Coene, G., Temmerman, M., Degomme, O., "Couple based family planning education: changes in male involvement and contraceptive use among married couples in Jimma Zone, Ethiopia”, BMC Public Health, vol. 15, pp. 682, 2015. DOI 10.1186/s12889-015-2057-y.

[3] FHI 360, "Increasing Men’s Engagement to Improve Family Planning Programs in South Asia”, 2012.

[4] Bruce, P., "The Involvement of Men in Family Planning: A Case of 37 Military Hospital", MPhil Thesis, University of Ghana, 2013.

[5] Vouking, MZ., Evina, CD., Tadenfok, CN., "Male involvement in family planning decision making in sub-Saharan Africa- what the evidence suggests”, Pan African Medical Journal, vol. 19, pp. 349, 2014. doi:10.11604/pamj.

[6] Wulifan, JK., Bagah, DA., "Male Involvement in Family Planning in Muslim Communities in Wa Municipality, Ghana”, Research on Humanities and Social Sciences, vol/issue: 5(7), 2015.

[7] Kabagenyi, A., Jennings, L., Reid, A., Nalwadda, G., Ntozi, J., Atuyambe, L., "Barriers to male involvement in contraceptive uptake and reproductive health services: a qualitative study of men and women's perceptions in two rural districts in Uganda”, Reproductive Health, vol. 11, pp. 21, 2014.

[8] Kassa, M., Alemu, A., Gedefaw, M., "Level of male involvement and associated factors in family planning services utilization among married men in Debremarkos town, Northwest Ethiopia”, BMC International Health and Human Rights, vol. 14, pp. 33, 2014.

[9] Walle, Y., Alamrew, Z., "The Current States of Male Involvement on Family Planning and Factors Correlated with among Male Factory Workers in Bahir Dar City”, American Journal of Public Health Research, vol/issue: 2(5), pp. 188-197, 2014. doi: 10.12691/ajphr-2-5-2.

[10] Program for Appropriate Technology in Health (PATH), “Involving Men in Reproductive Health”, vol/issue: 14(3), 1997.

[11] Steinfeld, EL., Newmann, SJ., Onono, M., Cohen, CR., Bukusi, EA., Grossman, D., “Overcoming Barriers to Family Planning through Integration: Perspectives of HIV-Positive Men in Nyanza Province, Kenya, Hindawi Publishing Corporation”, AIDS Research and Treatment, 2013. http://dx.doi.org/10.1155/2013/861983.

[12] Gizaw, A., Regassa, R., "Family planning service utilization in Mojo town, Ethiopia: A population based study", Journal of Geography and Regional Planning, vol/issue: 4(6), pp. 355-363, 2011.

[13] Adera, A., Belete, T., Gebru, A., Hagos, A., Gebregziabher, W., "Assessment of the Role of Men in Family Planning Utilization at Edaga-Hamuse Town, Tigray, North Ethiopia”, American Journal of Nursing Science, vol/issue: 4(4), pp. 174-181, 2015. doi: 10.11648/j.ajns.20150404.15.

[14] Ochako, R., Mbondo, M., Aloo, S., Kaimenyi, S., Thompson, R., Temmerman, M., Kays, M., "Barriers to modern contraceptive methods uptake among young women in Kenya: a qualitative study”, BMC Public Health, vol. 15, pp. 118, 2015. DOI 10.1186/s12889-015-1483-1.

[15] Central Statistics Agency of Federal Democratic Republic of Ethiopia, "Summary and Statistical Report of the 2007 Population and Housing Census Results”, Addis Ababa, 2007.

[16] Samuel, K., "Obstacles to Male Participation in Family Planning: A Study In Kiyeyi - Tororo District”, Makerere University, 2001. 